How to reference this article Słapek, D. (2019). Sulla declinazione dei cognomi italiani in polacco. Italica Wratislaviensia, 10(1), 233-259.

DOI: http://dx.doi.org/10.15804/IW.2019.10.1.10

\author{
Daniel Słapek \\ Uniwersytet Wrocławski \\ daniel.slapek@uwr.edu.pl, ORCID: 0000-0002-3755-9778
}

\title{
SULLA DECLINAZIONE DEI COGNOMI ITALIANI IN POLACCO
}

\section{ON THE DECLENSION OF ITALIAN SURNAMES IN POLISH}

\begin{abstract}
Polish inflexional morphology, with its rich inventory of rules relating to noun declension, also involves surnames of foreign origin. Generally, these surnames should be inflected just as Polish common names or adjectives are, depending on the inflexional paradigm to which they belong. However, the declension of foreign nouns involves various complications of phonetic, orthographic, and, most of all, inflexional natures, not only because of a relatively high number of rules governing the Polish morphological system, but also because some of these rules have not yet been determined in a definitive manner and the declension itself, in some cases, is optional. For this reason, speakers of Polish, to avoid inflecting a given surname, often prefer to adopt various editorial strategies, so to speak; in their texts, however, we can still find numerous incorrect inflexional forms and inconsistent or outright wrong inflexional strategies. With this article, then, I intend to expose the detailed rules relating to the declension of Italian surnames in Polish and analyse the occurrences of these surnames in Polish texts that have appeared in Italica Wratislaviensia.
\end{abstract}

Keywords: inflexion, declension, proper nouns, surnames, Italian surnames in Polish 


\section{INTRODUZIONE}

T a lingua polacca si caratterizza per un ricco sistema flessivo che coinvolge anche i nomi propri - tra cui quelli di provenienza straniera - che vanno declinati, tra l'altro, a seconda del caso ${ }^{1}$. I cognomi stranieri comportano a tal proposito particolari difficoltà, tanto che la norma linguistica permette in alcuni casi di ricorrere alla forma del nominativo se il cognome è accompagnato dal nome del suo portatore o da un nome comune il cui caso grammaticale aiuta a identificare la funzione logica dell'intero sintagma. Nell'uso corrente della lingua, infatti, s'incontrano spesso le forme invariate dei cognomi nei cosiddetti casi dipendenti ( $\mathrm{pl}$. przypadki zależne), ovvero diversi dal nominativo, anche laddove una simile forma non è prevista dalla norma grammaticale polacca ${ }^{2}$.

I problemi relativi alla flessione dei cognomi stranieri sono: 1) di natura ortografica: nella maggior parte dei casi, i cognomi stranieri mantengono la loro ortografia originale, tranne quelli che per tradizione si usano solo nella variante polonizzata, p. es. Cyceron da Cicero, o quelli che hanno due forme alternative, come p. es. Shakespeare/Szekspir o, per l'italiano, Petrarca/Petrarka (cf. Grzenia, 2002, p, 21)3); inoltre, alcuni segni diacritici di cui è privo l'alfabeto polacco possono essere semplificati nelle scritture meno formali, come $c ̧, \check{r}, \hat{\imath}$ ecc. (ivi, p. 22; ma non è il caso dei cognomi italiani); 2) di natura fonetica: come le alternanze consonantiche tipiche della lingua polacca, p. es. nom. Canaletto $>$ loc. Canaletcie, ma anche le "presunte" alternanze fonetiche che richiedono il cambiamento ortografico nei cognomi stranieri, p. es. nom. Vico > str. Vikiem (l'ortografia viene cambiata per evitare un'eventuale articolazione palatalizzata [t6] della consonante velare [k]); inoltre, la scorretta pronuncia di un segno ortografico straniero potrebbe contri-

1 Il sistema polacco dispone di 7 casi: nominativo, genitivo, dativo, accusativo, strumentale, locativo e vocativo; nel testo si useranno anche le loro seguenti abbreviazioni: nom., gen., dat., acc., str., loc. e voc.

2 Purtroppo, anche i cognomi polacchi rimangono spesso invariati, perfino nei testi formali (a questo proposito si veda p. es. Bajerowa, 1998).

3 Per esempio, nei saggi redatti in lingua polacca apparsi su Italica Wratislaviensia ricorre soltanto la variante polonizzata Petrarka. 
buire al cambiamento del paradigma flessivo, p. es. il cognome italiano Monza, se pronunciato "alla polacca" con una consonante fricativa $[\mathrm{z}]$ al posto di un'affricata, prenderebbe al dativo e al locutivo la desinenza grammaticale -e (*Monzie) invece di -y (Monzy); e - soprattutto - 3) di natura flessiva, dato un vasto repertorio di desinenze che offre il sistema morfologico polacco insieme alle complicate regole che lo governano.

Per via di una così complessa morfologia flessiva, la giusta declinazione dei cognomi stranieri in polacco non di rado richiede una certa consapevolezza linguistica del parlante e, quindi, gode di un certo prestigio sociale (la correttezza linguistica è a volte addirittura sopravvalutata giacché le viene attribuito un valore superiore a quanto effettivamente necessario per una semplice comunicazione; Bartmińska \& Bartmiński, 1992, p. 12). D'altro lato, la mancata o scorretta declinazione di un cognome, specie nei testi che richiedono un'edizione più accurata, è vista in maniera del tutto negativa (ricordiamo il titolo di un comunicato del Consiglio della Lingua Polacca istituito presso l'Accademia Polacca delle Scienze: "Vergogna a chi non declina i cognomi"; titolo originale: "Wstyd nie odmieniać nazwisk"; Bajerowa, 1998).

Con il presente lavoro intendo, quindi, esporre le regole relative alla declinazione dei cognomi italiani in polacco insieme a un'analisi delle occorrenze di tali cognomi nelle pagine di Italica Wratislaviensia (volumi 1-7, pubblicati tra il 2010 e il 2016) $)^{4}$. Va sottolineato che non si tratta di una semplice compilazione delle norme precedentemente raccolte, ma di una vera e propria revisione dei paradigmi flessivi munita di appositi commenti, dedicata a tutti coloro che operano tra la lingua italiana e polacca e che, nella loro esperienza quotidiana o professionale, si trovano di fronte al problema della flessione dei cognomi italiani in polacco.

4 Il titolo della rivista verrà indicato anche con l'abbreviazione "IW", talvolta seguita dal numero del volume a cui mi riferisco. 


\section{MODELLI DI DECLINAZIONE POLACCA DEI COGNOMI ITALIANI}

Secondo "le raccomandazioni generali" del Grande dizionario ortografico polacco - Wielki Stownik Ortograficzny PWN z zasadami pisowni i interpunkcji curato da Edward Polański -, i cognomi stranieri andrebbero declinati qualora possano inquadrarsi in uno dei paradigmi flessivi del sistema morfologico polacco. La scelta del paradigma dipende da tre fattori: 1) il sesso di chi porta il cognome, 2) la sua nazionalità, 3) la terminazione del lessema: si tratta sia dell'ultimo fonema di un dato cognome sia del fonema finale della sua radice (Polański, 2016, p. 96; similmente in Grzenia, 2002, p. 62, dove - per il terzo criterio - si preferiscono i termini "base grafica/fonetica").

Quanto alla terminazione del (cog)nome, in polacco si distinguono tre gruppi di consonanti: a) consonanti molli, che si pronunciano con un innalzamento della parte centrale del dorso della lingua verso il palato duro; queste sono: [z], [dz], [6], [t6], [n], cui va aggiunta l'approssimante palatale [j] (i suoni italiani che andrebbero considerati molli sono, quindi, i suoni palatali $[j],[n]$, nonché $[K]$ - assente in polacco - che in maniera semplificata viene pronunciato come $[\mathrm{lj}]$, quindi sempre con un suono molle $)$; b) consonanti dure, pronunciate senza un simile innalzamento del dorso della lingua: $[\mathrm{p}],[\mathrm{b}],[\mathrm{t}],[\mathrm{d}],[\mathrm{f}],[\mathrm{v}],[\mathrm{s}],[\mathrm{z}],[\mathrm{m}],[\mathrm{n}]$, [r], [1], cui va aggiunta l'approssimante palatale [w]; c) consonanti funzionalmente molli ${ }^{6}$ (dette anche storicamente molli o indurite), distinte all'interno del sistema consonantico polacco non perché abbiano una diversa natura fonetica, ma per il ruolo che svolgono dal punto di vista

5 Si vedano p. es. le entrate lessicali 'Badoglio' e 'Fenoglio' e le rispettive pronunce "badolio", "fenolio" nel dizionario di Bartmińska e Bartmiński (1992, pp. 32 e 80).

${ }^{6}$ Si useranno gli equivalenti italiani dei termini grammaticali polacchi comunemente approvati sia nei manuali di lingua polacca destinati ad apprendenti italiani (cf. p. es. Liotta, 1992, p. 8) sia nei dizionari di lingua polacca pubblicati in Italia (cf. Nosilia, 2017, p. 477). 
morfologico (cf. Gruszczyński \& Bralczyk, 2002, p. 267), tra le quali [ts], [dz], [J], [t]], [3], [dz]

È lecito notare che la pronuncia dei suoni trascritti, secondo l'alfabeto fonetico internazionale ${ }^{8}$, con i simboli [f], [tf], [dz] differisce nelle due lingue per quanto riguarda il loro punto di articolazione: in polacco hanno una pronuncia alveolare (cf. p. es. Ostaszewska \& Tambor, 2004, p. 40), in italiano invece sono pronunciati come prepalatali (cf. Maturi, 2006, p. 77) ${ }^{9}$ perciò andrebbero considerati - nella morfologia flessiva polacca - molli (la differenza nella pronuncia potrebbe provocare il cambiamento del paradigma flessivo tra i modelli $2 c$ e $2 d-$ molle $v s$ funzionalmente molle - presentati in seguito). In realtà, la pronuncia semplificata dei suoni italiani in polacco richiede che le tre consonanti prepalatali vengano accompagnate da una $[j]$, quindi i cognomi uscenti in italiano in -cia, -gia e -scia (dove - $i$ - non è accentata) in polacco abbiano comunque un suono consonantico finale molle (perché pronunciati come [t $\left.\left.\int j a\right],[d z j a],\left[\int j a\right]\right)^{10}$.

Tra le principali fonti delle norme relative alla declinazione dei cognomi (non solo) stranieri troviamo il già citato Wielki stownik ortograficzny $P W N$ z zasadami pisowni i interpunkcji (in questa sede mi riferisco all'edizione del 2016, pp. 93-107; la prima edizione è del 2003) e Wielki słownik poprawnej polszczyzny [Grande dizionario di

7 L'inventario delle consonanti indurite può variare a seconda dell'autore, p. es. Encyklopedia językoznawstwa ogólnego [Enciclopedia di linguistica generale] ne conta 6, come presentato sopra (Polański, 2003, p. 552), a cui invece Stownik gramatyki języka polskiego [Dizionario di grammatica della lingua polacca] aggiunge la consonsante [1] (Gruszczyński \& Bralczyk, 2002, p. 267); per le nostre considerazioni [1] verrà classificata come dura, altrimenti le desinenze di alcuni cognomi potrebbero essere confuse tra i modelli di declinazione $3 c$ e $3 d$ esposti in seguito.

8 Per la trascrizione dei suoni polacchi secondo l'alfabeto fonetico internazionale si veda Miodunka, 1977, pp. xxiv-xxvi.

9 Nell'unico manuale italo-polacco dedicato alla grammatica contrastiva si preferisce una classificazione semplificata, dove i suoni [ $\left.\int\right],\left[\mathrm{t} \int\right],[\mathrm{d} 3]$ sono considerati prepalatali in entrambe le lingue (Kwapisz-Osadnik, 2012, p. 19). La differenza tra questi suoni è stata giustamente notata da Olga Broniś (2017, p. 188).

10 Si veda p. es. l'entrata lessicale 'Borgia': “bordżja” (Grzenia, 2002, p. 109) o "bordżia” (Bartmińska \& Bartmiński, 1992, p. 44; Cieślikowa, 2008, p. 149). 
lingua polacca corretta] curato da Andrzej Markowski (il capitolo dedicato ai cognomi è di Dorota Zdunkiewicz-Jedynak, 2006; $1^{\text {a }}$ ed. del 2004). Inoltre, nell'offerta editoriale polacca sono apparse varie pubblicazioni dedicate alla cosiddetta cultura linguistica, come p. es. Kultura języka polskiego [Cultura della lingua polacca] di Hanna Jadacka (2005), o numerosi dizionari interamente dedicati ai nomi propri, tra i quali Slownik wymowy i odmiany nazwisk obcych [Dizionario di pronuncia e di declinazione dei cognomi stranieri] di Izabella Bartmińska e Jerzy Bartmiński (1992), Stownik nazw własnych [Dizionario di nomi propri] di Jan Grzenia (2002; $1^{a}$ ed. del 1998) e Mały słownik odmiany nazw własnych [Piccolo dizionario di declinazione dei nomi propri] curato da Aleksandra Cieślikowa (2008, $1^{\mathrm{a}}$ ed. del 2002).

I titoli appena citati presentano i modelli flessivi dei cognomi stranieri in maniera diversa quanto alla precisione, per così dire, delle norme che determinano e - soprattutto - non sono dedicati specificamente alla declinazione dei cognomi italiani. Per questo motivo cambia il numero di modelli presi in considerazione (alcuni autori ammettono expressis verbis che si tratta di una semplificazione; cf. Bartmińska \& Bartmiński, 1992, p. 21), cambiano anche i criteri di categorizzazione dei paradigmi, tra cui il suono finale (Grzenia, Jadacka) e la flessione sostantivale/ aggettivale (Zdunkiewicz-Jedynak). L'unico titolo che divide i modelli anche a seconda della provenienza nazionale del cognome è il dizionario di Polański, ma il capitolo dedicato ai cognomi italiani indica solo 3 gruppi, accompagnati da informazioni piuttosto sommarie (2016, p. 101; regole 255-257). Nelle pagine che seguono cercherò, quindi, di esporre in maniera quanto più possibile dettagliata i modelli flessivi che si addicono ai cognomi italiani. I paradigmi che vedremo sono privi del caso vocativo. Infatti, in polacco la forma dei cognomi al vocativo, anche se possibile, è estremamente rara perché l'uso del cognome nei riferimenti diretti all'interlocutore è culturalmente considerato sgarbato (cf. Zdunkiewicz-Jedynak, 2006, p. 1622). Inoltre, nelle tabelle 1 e 2 i casi che hanno le forme flesse coincidenti sono riportati in una stessa riga. 


\subsection{Cognomi maschili variabili}

In linea di massima, $\mathrm{i}$ cognomi che terminano con una consonante o con le vocali $-a,-o$ si declinano in polacco come nomi comuni, invece i cognomi uscenti in $-i$, $-e$ hanno una declinazione aggettivale (ivi, p. 1612 $)^{11}$. In tal modo, per i cognomi italiani si possono distinguere i seguenti modelli di declinazione ${ }^{12}$ :

1) I cognomi che terminano con una consonante o con la vocale $-o^{13}$ (non accentata) seguono la declinazione sostantivale maschile personale; in questo gruppo possiamo indicare tre varianti del paradigma flessivo a seconda del tipo di consonante che termina la radice lessicale di un dato cognome:

1a) quando la consonante finale è dura (eccetto $[\mathrm{k}], g$ e $l$ ) i cognomi si declinano come il nome comune profesor, p. es. nom. Bruno, gen. Bruna, dat. Brunowi, acc. Bruna, str. Brunem, loc. Brunie; per i nomi uscenti in $-o$ preceduta da una doppia consonante dura, al locativo viene palatalizzato il secondo elemento del nesso consonantico che, tuttavia, nella pronuncia non subisce l'assimilazione regressiva, p. es. nom. Canaletto, loc. Canaletcie (cf. Zdunkiewicz-Jedynak, 2006, p. 1615);

1b) quando la consonante finale è molle, funzionalmente molle o $l$, i cognomi seguono l'esempio di piekarz: nom. Pirandello, gen. Pirandella, dat. Pirandellowi, acc. Pirandella, str. Pirandellem, loc. Pirandellu (cambiano, quindi, la desinenza del locativo rispetto al modello $1 a$ );

11 Laddove l'ortografia corrisponde alla trascrizione fonetica, i suoni verranno indicati in corsivo, altrimenti riporterò tra parentesi quadre i simboli dell'API, visto che si tratta di suoni. Quando nel testo si parla del suono [k], si tratta solo della corrispondente lettera $c$ e non del digramma $c h$, come in Boschi; invece, quando si parla della lettera $g$, si tratta solo del suono [g] e non [dz].

12 Tutti gli esempi dei cognomi italiani qui riportati sono facilmente ritrovabili nei vari dizionari specialistici, come il Dizionario dei cognomi italiani di Emidio de Felice (1978) o il Dizionario etimologico-semantico dei cognomi italiani di Mario Alinei e Francesco Benozzo (2017).

13 Si parla a questo proposito dei cognomi non slavi (Grzenia, 2002, p. 67, Zdunkiewicz-Jedynak, 2006, p. 1615); i cognomi slavi uscenti in -o seguono la flessione femminile. 
1c) quando la consolante finale è velare, allo strumentale va aggiunta la vocale $i$ davanti alla desinenza -em (nom. Longo, str. Longiem); inoltre, i cognomi il cui suono consonantico finale è [k], quindi in italiano reso ortograficamente con una $c$, allo strumentale cambiano l'ortografia in quella polacca, p. es. nom. Fusco, str. Fuskiem (cf. ibidem).

Per il primo gruppo si possono indicare alcuni cognomi tradizionalmente invariabili, tra cui - quanto ai cognomi italiani - Eco (Grzenia, 2002 , p. 67) ${ }^{14}$, ma la sua flessione non è ancora stabilita poiché non è difficile ritrovare i testi - anche di natura formale - dove il detto cognome viene declinato ${ }^{15}$.

2) I cognomi uscenti in - $a$ seguono la declinazione sostantivale femminile con alcune variazioni a seconda del suono finale:

2a) quando la vocale - $a$ è preceduta da una consonante dura (eccetto $[\mathrm{k}], g, l)$, i cognomi si declinano come rama, p. es. nom. Scarpa, gen. Scarpy, dat. Scarpie, acc. Scarpe, str. Scarpa, loc. Scarpie; similmente al modello $1 a$, se la desinenza è preceduta da una doppia consonante dura, al locativo viene palatalizzata solo la seconda consonante e l'intero nesso non viene semplificato nella pronuncia, p. es. nom. Gambetta, loc. Gambetcie (cf. Zdunkiewicz-Jedynak, 2006, p. 1617);

14 Tutti i dizionari dei nomi propri cui si fa riferimento in questa sede riportano il cognome Eco come invariabile (Bartmińska \& Bartmiński, 1992, p. 77; Cieślikowa, 2008, p. 169; Grzenia, 2002, p. 149).

15 Si veda p. es. il saggio di Roman Konik, "Katastrofiści i przystosowani czyli typologia współczesnych intelektualistów według Umberta Eca" (2003), dove la forma declinata appare nello stesso titolo; tuttavia, è lecito notare che l'autore preferisce al dativo la forma invariata accompagnata dal nome: Umbertowi Eco (ivi, p. 276); nel saggio Eco è stato declinato solo al genitivo e all'accusativo, non ricorrono le forme dello strumentale Ekiem (per via del cambiamento ortografico - obbligatorio in questo caso - tale forma risulterebbe, forse, difficile da riconoscere) né del locativo $E c u$ (similmente, la forma $E c a$ del gen./acc. ricorre in Wojtowicz 1997; a proposito della declinazione di Eco in IW si veda la sezione 3.2). È, inoltre, interessante citare la comunicazione apparsa sul sito della consulenza linguistica della Scuola Superiore della Bassa Slesia [Dolnośląska Szkoła Wyższa], dove la forma declinata Eca viene chiamata "la più corretta" (Katedra Kulturoznawstwa DSW, n.d.; sul sito non appare il nome dell'autore). 
2b) quando la vocale - $a$ è preceduta da una consonante velare, i cognomi si declinano come noga, p. es. nom. Ferluga, gen. Ferlugi, dat. Ferludze, acc. Ferluge, str. Ferluga, loc. Ferludze; similmente al modello $1 c$, i cognomi il cui suono consonantico finale è $[\mathrm{k}](c)$, al genitivo cambiano l'ortografia, p. es. nom. Petrarca, str. Petrarki ${ }^{16}$;

2c) quando la vocale - $a$ è preceduta da una consonante molle o $l$, la declinazione segue l'esempio di dynia: nom. Battaglia, gen. Battagli, dat. Battagli, acc. Battaglie, str. Battaglia, loc. Batta$g l i$; come si è notato prima, a questo gruppo appartengono anche le terminazioni italiane con una consonante prepalatale [S], [t $\mathrm{f}]$, [d3] seguita da -ia, dove $i$ in polacco viene pronunciata come approssimante [j]; inoltre, $i$ tre suoni prepalatali italiani richiedono il raddoppiamento della vocale davanti alla desinenza $-i$ : nom. Borgia, Quercia, Sciascia, gen./dat./loc. Borgii, Quercii, Sciascii (cf. p. es. Bartmińska \& Bartmiński, 1992, pp. 44, 160, $179)^{17}$

2d) quando la vocale - $a$ è preceduta da una consonante funzionalmente molle, i cognomi si declinano come róża, p. es. nom. Lanza, gen. Lanzy, dat. Lanzy, acc. Lanze, str. Lanza, loc. Lanzy, quindi rispetto al modello $2 c$ cambiano la $-i$ del gen./dat./loc. in $-y$.

3) I cognomi uscenti in -i si declinano come aggettivi maschili polacchi la cui consonante finale è molle, come tani, p. es. non. Verdi, gen. Verdiego, dat. Verdiemu, acc. Verdiego, str. Verdim, loc. Verdim; i nomi uscenti in -li, davanti alle desinenze -ego/-emu, perdono la $i$, p. es. nom. Botticelli, gen. Botticellego (cf. Polański, 2016, p. 101); va notato che la desinenza -im dello strumentale e del locativo non comporta il raddoppiamento della $i$ finale del cognome, quindi loc. Seriannim e non *Serianniim (cf. Grzenia, 2002, p. 66).

16 Le fonti citate non propongono una regola esplicita a questo proposito, tuttavia è facile dedurla da vari esempi ritrovati nei dizionari di "lingua corretta".

17 Anche in questo caso nelle fonti citate non troveremo una regola precisa (cf. nota 16). 
4) I cognomi uscenti in -e si declinano come aggettivi neutri (fanno eccezione lo strumentale e il locativo, per i quali casi prendono la desinenza -em e non -ym), p. es. nom. Pavese, gen. Pavesego, dat. Pavesemu, acc. Pavesego, str. Pavesem, loc. Pavesem.

\subsection{Cognomi maschili invariabili}

Rimangono invariati 1) i cognomi uscenti in $-u$ (la quale vocale, nei cognomi polacchi, ricorre solo all'inizio o all'interno della parola; cf. Jadacka, 2005, p. 54; Grzenia, 2002, p. 65), p. es. Careddu; nonché 2) i cognomi che finiscono con la vocale -o accentata (ibidem), p. es. Calabrò (a questa categoria appartengono anche i monosillabi quali $B o$, Fo); quanto alle parole tronche, si parla solo della vocale -o, per cui i cognomi che finiscono con altre vocali accentate vanno sottoposti alla declinazione ${ }^{18}$, tranne quelli uscenti in $-\grave{u}$ (p. es. Chiuchiù) per motivi di cui sopra; inoltre, l'accento grafico nelle forme diverse dal nominativo può essere omesso (ivi, p. 66) ${ }^{19}$.

Quanto alle forme invariabili, si parla anche dei cognomi il cui suono finale è assente in lingua polacca, come in Dubois, quindi [wa] (Jadacka, 2005, p. 54; Grzenia, 2002, p. 65), ma - a quanto pare - si tratta solo dei cognomi di provenienza francese (Grzenia riporta tra gli esempi il cognome Pasqua, ma sempre nella versione francese; ivi, p. 65) ${ }^{20}$. Tale categoria non inquadra, quindi, i cognomi italiani giacché la consonante palatale $[K]$ è sempre seguita da una vocale, ed è comunque

18 In italiano, infatti, i cognomi possono terminare con tutte le vocali accentate, p. es. Cannistrà, Vicerè, Macrì.

${ }_{19}$ Grzenia dà sempre l'esempio di un cognome francese: nom. Fuaré, gen. Fuarégo o Fuarego (ibidem), ma tale strategia ortografica va adoperata anche per i cognomi italiani; si paragoni p. es. la traduzione polacca de I dubbi di Salaì di Rita Monaldi e Francesco Sorti, dove la versione declinata non porta l'accento: "Wątpliwości Salaiego" (traduzione di Justyna Łukaszewicz).

${ }^{20}$ Sul sito della consulenza linguistica dell'editore PWN si legge la risposta dell'autore al quesito relativo alla declinazione del cognome del politico francese Charles Pasqua (Grzenia, 2007); simili cognomi italiani non comportano alcuna difficoltà flessiva. 
semplificata in polacco in [1j] (chiaramente, anche le vocali chiuse [o] ed $[\mathrm{e}]$ in polacco si pronunceranno come aperte).

Un caso particolare dell'invariabilità flessiva sono $\mathrm{i}$ cognomi che terminano in - $a u$ [aw] perché ne rimane invariato solo il locativo. Altre forme flesse aggiungono adeguate desinenze del paradigma maschile con una consonante finale dura, seguono quindi il modello la (ne fa nota solo il dizionario di Grzenia, ivi, p. 68). Tra i cognomi italiani che potrebbero rispettare questa regola troviamo Cau (il cognome sardo che ricorre anche tra le pagine di IW, si veda il seguito), ma la pronuncia italiana è $[\mathrm{au}]$ - e non con l'approssimante [aw] -, per cui è giusto considerarlo come non variabile ${ }^{21}$.

Sono, inoltre, accettabili le forme invariate dei cognomi uscenti in -o, - e o che terminano con una sillaba accentata, ma in tal caso vanno preceduti da un nome comune (p. es. un tecnonome, come adwokat, profesor ecc.) o dal nome del portatore dello stesso cognome che è declinato e che potrà indicare la funzione logica dell'intero sintagma (Grzenia, 2002 p. 65; Polański, 2016, p. 97) ${ }^{22}$. In alcuni casi, per motivi stilistici o per la necessità comunicativa di citare la forma del nominativo, talvolta difficilmente ricostruibile dai casi dipendenti (p. es. dei cognomi ossitoni che portano l'accento grafico), tale scelta è del tutto raccomandabile.

21 Inoltre, se pronunciato [kaw], corrisponde in polacco a 'feci', per cui sarebbe - senza alcun dubbio - consigliabile ricorrere alla forma invariata del cognome preceduta dal nome. In realtà, i suoni [wa] e [au] sono presenti in polacco (p. es. auto [auto], tawa [wava]; cf. Karaś \& Madejowa, 1977, pp. 13, 187); gli autori si riferiscono probabilmente alla terminazione dei cognomi francesi la cui pronuncia non è identica a quella polacca.

22 Tra i cognomi che dimostrano una simile tendenza Zdunkiewicz-Jedynak registra anche i cognomi uscenti in - $i$ (2006, p. 1620); ne risulta che - visti i paradigmi flessivi esposti sopra - tutti i cognomi italiani che terminano con una consonante o con la vocale - $a$ vanno declinati. 
Tabella 1: Declinazione dei cognomi italiani maschili in polacco

\section{Cognomi variabili}

\section{Cognomi che terminano con una consonante o con la vocale -o non accentata}

1a) (-o preceduta da) consonante dura (tranne $[\mathrm{k}], g, l$ )

\begin{tabular}{l|l|l|l}
\hline nom. & De Amicis & Cagliostro & Canaletto \\
\hline gen./acc. & De Amicisa & Cagliostra & Canaletta \\
\hline dat. & De Amicisowi & Cagliostrowi & Canalettowi \\
\hline str. & De Amicisem & Cagliostrem & Canalettem \\
\hline loc. & De Amicisie & Cagliostrze & Canaletcie \\
\hline $\begin{array}{l}\text { 1b) }(-o \text { preceduta da) consonante } \\
\text { molle o } l\end{array}$ & molle, funzionalmente & $\begin{array}{l}\text { c) }[\mathrm{k}] \text { o } g \text { (seguita } \\
\text { da o) }\end{array}$ \\
\hline nom. & Pirandello & Boccaccio & Fusco \\
\hline gen./acc. & Pirandella & Boccaccia & Fusca \\
\hline dat. & Pirandellowi & Boccacciowi & Fuscowi \\
\hline str. & Pirandellem & Boccacciem & Fuskiem \\
\hline loc. & Pirandellu & Boccacciu & Fuscu \\
\hline
\end{tabular}

2. Cognomi che terminano in $-a$

2a) - $a$ preceduta da una consonante dura (tranne $[\mathrm{k}], g, l$ )

\begin{tabular}{l|l|l|l}
\hline nom. & Scarpa & Costa & Gambetta \\
\hline gen. & Scarpy & Costy & Gambetty \\
\hline dat./loc. & Scarpie & Coście & Gambetcie \\
\hline acc. & Scarpę & Costę & Gambettę \\
\hline str. & Scarpą & Costą & Gambettą \\
\hline
\end{tabular}

2b) - $a$ preceduta da una consonante velare $[\mathrm{k}]$ o $g$

\begin{tabular}{l|l|l|l}
\hline nom. & Ferluga & Manca & Rocca \\
\hline gen. & Ferlugi & Manki & Rokki \\
\hline dat./loc. & Ferludze & Mance & Rocce \\
\hline acc. & Ferlugę & Mancę & Roccę \\
\hline str. & Ferlugą & Mancą & Roccą
\end{tabular}




\section{Cognomi variabili}

\begin{tabular}{l|l|l|l}
\hline \multicolumn{2}{c|}{ 2c) $-a$ preceduta da una consonante molle o $l$} & 2d) a $+[\mathrm{ts}]$ o $[\mathrm{dz}]$ \\
\hline nom. & Coppola & Borgia & Lanza \\
\hline gen./dat./loc. & Coppoli & Borgii & Lanzy \\
\hline acc. & Coppole & Borgię & Lanzę \\
\hline str. & Coppolą & Borgią & Lanzą \\
\hline \multicolumn{2}{r}{ 3) Cognomi che terminano in $-\boldsymbol{i}$} & 4) terminano in $\boldsymbol{e}$ \\
\hline nom. & Verdi & Botticelli & Pavese \\
\hline gen./acc. & Verdiego & Botticellego & Pavesego \\
\hline dat. & Verdiemu & Botticellemu & Pavesemu \\
\hline str./loc. & Verdim & Botticellim & Pavesem \\
\hline
\end{tabular}

II. Cognomi invariabili

\begin{tabular}{l|l|l|l}
\hline & $1)-u$ & $2)-\grave{o}(-o$ accentata $)$ & $\begin{array}{l}\text { 3) prep. (sopranno- } \\
\text { me })\end{array}$ \\
\hline n./g/d./a./s./l. & Careddu & Calabrò & da Vinci \\
\hline
\end{tabular}

consonanti dure: [p], [b], [t], [d], [f], [v], [s], [z], [m], [n], [r], [1] + [w] consonanti molli: [z], [dz], [6], [t6], [n] + [j] consonanti funzionalmente molli: [ts], [dz], [J], [t $],[3],[\mathrm{d} 3]$

\subsection{Cognomi femminili}

La declinazione femminile è molto meno complessa: si declinano i cognomi italiani che terminano in $-a$; in tal caso seguono le stesse regole della declinazione dei cognomi stranieri maschili uscenti in - $a$ (come nella TABELLA 2). Altri cognomi rimangono invariati. 
TABella 2: Declinazione dei cognomi italiani femminili uscenti in $-a$ in polacco

\begin{tabular}{|c|c|c|c|c|c|}
\hline & $\begin{array}{c}\text { cons. dura } \\
\text { tranne }[\mathrm{k}], g, l\end{array}$ & consonar & elare $[\mathrm{k}] \mathrm{og}$ & $\begin{array}{c}\text { consonante molle } \\
0 l\end{array}$ & $\begin{array}{l}\text { c. funzional- } \\
\text { mente molle: }\end{array}$ \\
\hline nom. & Deledda & Marlega & Rusca & Campiglia & Sforza \\
\hline gen. & Deleddy & Marlegi & Ruski & Campigli & Sforzy \\
\hline dat./loc. & Deleddzie & Marledze & Rusce & Campigli (=gen.) & Sforzy (=gen.) \\
\hline acc. & Deleddę & Marlegę & Ruscę & Campiglię & Sforzę \\
\hline str. & Deleddą & Marlegą & Ruscą & Campiglią & Sforzą \\
\hline
\end{tabular}

\subsection{COGNOMI COMPOSTI}

Nei cognomi composti ogni componente si declina come un cognome semplice, per cui segue tutte le regole sopra esposte (cf. ZdunkiewiczJedynak, 2006, p. 1620)

\section{COGNOMI ITALIANI NEI TESTI POLACCHI APPARSI SU ITALICA WRATISLAVIENSIA}

Nei primi sette volumi di Italica Wratislaviensia sono apparsi numerosi testi redatti in lingua polacca (35 saggi su un totale di 84$)^{23}$ in cui ricorrono 744 forme testuali diverse dal nominativo di 234 cognomi italiani ${ }^{24}$. La stragrande maggioranza dei cognomi - 624 parole testua-

23 Dal vol. 8 (2017), visti i criteri di parametrizzazione delle riviste scientifiche e l'importanza che viene attribuita alle cosiddette lingue da congresso (tra cui francese, inglese, italiano, tedesco, russo e spagnolo), la redazione di IW - per facilitare il riconoscimento ufficiale della rivista e, quindi, per dare agli autori uno strumento di diffusione delle ricerche altamente classificato - ha deciso di accettare testi redatti soltanto in lingua italiana.

${ }^{24}$ Non sono stati presi in considerazione i cognomi a) le cui forme sono polonizzate, come p. es. gen. Petrarki, dat. Petrarce (IW1, p. 24, dove la forma del nominativo adoperata dall'autore è Petrarka), gen. Baroniusza [Cezarego] (IW5, p. 28, da 'Cesare Baroni') o gen. Bosko [Jana] (IW6, p. 264; da 'Giovanni Melchiorre Bosco'; la versione polacca è rimasta - curiosamente - invariata all'accusativo), 
li - sono forme declinate; 56 occorrenze sono i cognomi invariabili (54 forme dei cognomi femminili e 2 forme dei cognomi maschili; per una statistica dettagliata si veda nell'appendice la TABELLA 4$)^{25}$. Inoltre, nei testi polacchi troveremo 64 forme testuali di cognomi potenzialmente variabili che non sono state declinate, tra cui 31 forme del cognome Eco e 4 forme di Cau (che si caratterizzano per un'incerta appartenenza al paradigma flessivo; si veda più avanti in $\S 3.3$ ), quindi 29 occorrenze che potrebbero risultare interessanti per la presente analisi e che sono esposte nella tabella $3^{26}$.

TABeLla 3: Cognomi maschili potenzialmente variabili non declinati su IW

\begin{tabular}{c|c|c|c|c|c|c|c}
\hline vol. & p. & caso & $\begin{array}{c}\text { nome } \\
\text { (forma) }\end{array}$ & $\begin{array}{c}\text { cognome } \\
\text { (forma) }\end{array}$ & $\begin{array}{c}\text { cognome } \\
\text { (nom.) }\end{array}$ & $\begin{array}{c}\text { decl. } \\
\text { nome }\end{array}$ & prep. \\
\hline 1 & 21 & acc. & Giovanniego & Della Casa & Della Casa & 1 & 1 \\
\hline 1 & 17 & gen. & Francesca & da Barberino & da Barberino & 1 & 1 \\
\hline 1 & 17 & gen. & Antonia & da Tempo & da Tempo & 1 & 1 \\
\hline 1 & 57 & gen. & A. & Baricco & Baricco & - & 0 \\
\hline 1 & 57 & gen. & A. & de Carlo & de Carlo & - & 1 \\
\hline 1 & 59 & gen. & C. & Malaparte & Malaparte & - & 0 \\
\hline 1 & 113 & gen. & Roberta & Saviano & Saviano & 1 & 0 \\
\hline 1 & 119 & gen. & Matteo & Garrone & Garrone & 0 & 0 \\
\hline 1 & 119 & acc. & - & Saviano & Saviano & - & 0 \\
\hline
\end{tabular}

b) dei personaggi non italiani, anche se etimologicamente di provenienza italiana, come p. es. gen. Zanussiego [Krzysztofa] (IW5, p. 29), c) al plurale, p. es. gen.pl. Bembów (IW6, p. 100), d) presenti nelle citazioni prese da altri testi (si veda p. es. IW7 p. 116).

${ }^{25}$ Il file contenente tutte le forme flesse dei cognomi italiani ritrovati nelle pagine di IW insieme a una statistica complessiva è disponibile sul profilo academia.edu dell'autore.

${ }^{26}$ Le ultime due colonne della tabella sono dedicate 1) alla declinazione del nome che accompagna il cognome $(1$ - nome declinato, 0 - non declinato, trattino il nome non ricorre), e 2) alla presenza di una preposizione nella forma del cognome ( 1 - presente; 0 - non presente). 


\begin{tabular}{|c|c|c|c|c|c|c|c|}
\hline vol. & p. & caso & $\begin{array}{c}\text { nome } \\
\text { (forma) }\end{array}$ & $\begin{array}{l}\text { cognome } \\
\text { (forma) }\end{array}$ & $\begin{array}{l}\text { cognome } \\
\text { (Nom.) }\end{array}$ & $\begin{array}{l}\text { decl. } \\
\text { nome }\end{array}$ & prep. \\
\hline 1 & 120 & gen. & - & Saviano & Saviano & - & 0 \\
\hline 1 & 122 & gen. & - & Saviano & Saviano & - & 0 \\
\hline 1 & 151 & gen. & Leonarda & da Vinci & da Vinci & 1 & 1 \\
\hline 3 & 71 & gen. & Jacopona & da Todi & da Todi & 1 & 1 \\
\hline 3 & 68 & gen. & Carla & Felice & Felice & 1 & 0 \\
\hline 3 & 76 & acc. & Carla & Pisacane & Pisacane & 1 & 0 \\
\hline 3 & 76 & gen. & - & Pisacane & Pisacane & - & 0 \\
\hline 3 & 77 & loc. & - & Pellico & Pellico & - & 0 \\
\hline 5 & 30 & acc. & Vincenzia & da Parmataro & $\begin{array}{c}\text { da Parma- } \\
\text { taro }\end{array}$ & 1 & 1 \\
\hline 5 & 99 & gen. & Pietra & da Cortona & da Cortona & 1 & 1 \\
\hline 5 & 114 & str. & $\begin{array}{c}\text { Giovannim } \\
\text { Battistą }\end{array}$ & de Carlis & de Carlis & 1 & 1 \\
\hline 5 & 325 & gen. & Antonia & da Ponte & da Ponte & 1 & 1 \\
\hline 6 & 104 & gen. & Dionigiego & $\begin{array}{c}\text { da Borgo San } \\
\text { Sepolcro }\end{array}$ & $\begin{array}{c}\text { da Borgo } \\
\text { San Sepol- } \\
\text { cro }\end{array}$ & 1 & 1 \\
\hline 6 & 108 & gen. & Dionigiego & $\begin{array}{c}\text { da Borgo San } \\
\text { Sepolcro }\end{array}$ & $\begin{array}{c}\text { da Borgo } \\
\text { San Sepol- } \\
\text { cro }\end{array}$ & 1 & 1 \\
\hline 6 & 260 & gen. & Vittorina & da Feltre & da Feltre & 1 & 1 \\
\hline 6 & 268 & gen. & - & Lombroso & Lombroso & - & 0 \\
\hline 6 & 268 & gen. & - & Lombroso & Lombroso & - & 0 \\
\hline 6 & 268 & gen. & Cesarego & Lombroso & Lombroso & 1 & 0 \\
\hline 7 & 101 & gen. & Francesa & De Sanctis & De Sanctis & 1 & 1 \\
\hline 7 & 147 & gen. & - & Pavese & Pavese & - & 0 \\
\hline
\end{tabular}




\subsection{Osservazioni generali}

Gli autori di IW insieme alla redazione della rivista paiono particolarmente attenti alla declinazione dei cognomi: solo 12 occorrenze tra tutte le 688 forme potenzialmente variabili ${ }^{27}$ sono rimaste invariate e non precedute da un nome, quindi si tratta di uno scarso $1,74 \%$. Inoltre, tutti i cognomi italiani che terminano con una consonante o con le vocali $-a$ sono stati accuratamente declinati.

Come si è detto prima, i nomi uscenti in -o, ed -e possono rimanere invariati se accompagnati da un nome declinato. Sono, quindi, pienamente accettabili le forme: gen. Roberta Saviano, gen. Carla Felice, acc. Carla Pisacane e gen. Cesarego Lombardoso - quest'ultima particolarmente interessante, perché i nomi maschili stranieri che terminano in -e possono ugualmente non declinarsi in polacco (anche se si diffondono sempre di più le forme declinate; cf. Polański, p. 96).

Sono, invece, da considerarsi scorretti i sintagmi in cui rimangono invariati al genitivo sia il nome sia il cognome (1), oppure quelli in cui appare soltanto un cognome invariato (2-9). Inoltre, è particolarmente incoerente dal punto di vista stilistico la frase in cui cooccorrono più cognomi diversamente declinati (6).

(1) [...] widzowie mogli oglądać filmową adaptację Gomorry w reżyserii Matteo Garrone (IW1, p. 119)

(2) A ten inny świat, opisany przez Saviano, jest przerażający. (ivi, p. 119)

(3) Reporterska powieść Saviano była szokiem także dla Włochów [...] (ivi, p. 120)

(4) [...] książka Saviano przestała być postrzegana jako analiza [...] (ivi, p. 122)

(5) W wyprawie do Sapri, obok Pisacane i jego dwudziestu czterech towarzyszy [...] (IW3, p. 76)

(6) Jak już zauważyliśmy, wspominając o Aleardim czy Pellico [...] (ivi, p. 77)

(7) W jego opinii całość rozważań Lombroso można streścić [...] (IW6, p. 268)

2756 occorrenze dei cognomi invariabili sono state sottratte dal numero complessivo 744. In questa statistica sono inclusi i cognomi preceduti dalla preposizione $d a$ che indicano la provenienza del personaggio (ne parlerò in seguito). 
(8) Treść książki Lombroso przedstawiono na łamach [...] (ibidem)

(9) Dominujący w wierszu Pavese czas teraźniejszy okazuje się [...] (IW7, p. 147)

Le forme invariate precedute da un'iniziale del nome potrebbero essere accettate (soprattutto quando si tratta di scrittori i cui nomi sono comunemente riconosciuti), se tutta la frase fosse coerente dal punto di vista flessivo. Nei frammenti 10-11 alcuni cognomi in -o, invece, sono stati declinati (gen. Fogazzara, Berta) ${ }^{28}$.

(10) [...] autorka przekładów: A. Baricco, A. de Carlo, A. Fogazzara, C. E. Gaddy, A. Moravii, A. M. Ripellina, L. Sciascii, A. Tabucchiego [...] (IW1, p. 57)

(11) [...] przełożyła liczne utwory, m.in. G. Berta, I. Calvina, N. Ginzburg. A. Manzoniego, C. Malaparte, E. Vittoriniego. (ivi, p. 59)

3.2. Cognomi preceduti da una preposizione

Gli autori delle principali fonti relative alla flessione dei cognomi in polacco, citati in questa sede già varie volte, non hanno definito alcuna regola specifica concernente i cognomi stranieri preceduti da una preposizione. Si presume che essi vengano declinati come altri cognomi stranieri. Tuttavia, come nota Adam Wolański (2018), numerosi cognomi uscenti in $-e,-i,-o$ rimangono in pratica invariati perché la loro forma pare estranea al sistema linguistico polacco (altre desinenze andrebbero, in tal caso, declinate).

Quanto alla presente analisi di IW, il numero complessivo di occorrenze dei cognomi preceduti da una preposizione è 74. Sono state declinate tutte le 30 occorrenze di D'Annunzio (casi: gen./acc. D'Annunzia, str. D'Annunziem, loc. D'Annunziu), tutte le 27 occorrenze di De Amicis (casi: gen./acc. De Amicisa) e le singole occorrenze dei seguenti cognomi: De Sanctis (gen. De Sanctisa, IW7, p. 101), della Bella (gen. Della Belli, IW5, p. 99), Dall'Ongaro (gen. Dall'Ongara, IW3, p. 78), De Gasperi (gen. De Gasperiego, IW1, p. 91).

28 Va notato che entrambi i frammenti provengono dalle note a pie di pagina, quindi dai frammenti che sfuggono più facilmente nel corso della revisione linguistica. 
Quasi tutte le forme invariate (13 su 14) sono state accompagnate da un nome declinato (si tratta di cognomi: da Barberino, da Borgo San Sepolcro, da Cortona, da Feltre, da Parmataro, da Ponte, da Tempo, da Todi, da Vinci, de Carlis, de Carlo, De Sanctis, Della Casa; ne risulta che gli autori di IW hanno lasciato invariati tutti i cognomi preceduti dalla preposizione $d a$ ), un'occorrenza testuale, invece, dall'iniziale del nome: A. de Carlo (IW1, p. 57; in questo caso si preferirebbe una forma declinata del nome e del cognome $)^{29}$. Tra questi, potrebbero facilmente declinarsi i cognomi de Carlo (uscente in -o, quindi appartenente al paradigma 1a), de Carlis (cf. sopra: gen. De Amicisa; similmente, il paradigma $1 a$ ), si tratta quindi di una scelta personale dell'autore, accettabile, perché viene citato anche il nome del personaggio.

L'unico cognome preposizionale che registra sia la forma declinata sia quella invariata è De Sanctis, ma in questo caso l'autrice ricorre alla strategia stilistica "cognome invariato preceduto da un nome declinato / solo cognome declinato", come leggiamo nei frammenti (12-13).

(12) Na zakończenie wróćmy do krytyka Francesca De Sanctis, który [...] (IW7, p. 101)

(13) To, co było prawdą dla De Sanctisa w 1870 r., kiedy [...] (ibidem)

I cognomi preposizionali uscenti in - $a$, quindi potenzialmente variabili, sono due: Della Casa (cf. sopra gen. Della Belli) e Da Cortona. Si tratta, però, in entrambi i casi di soprannomi che non sono stati polonizzati (p. es. da Cortona $>$ pl. $z$ Cortony) ${ }^{30}$, e tali soprannomi non vanno declinati (Wolański, 2008).

(14) $[\ldots]$ schematy stosowane szeroko w szesnastowiecznej poezji włoskiej, np. przez Vittorię Colonnę, Pietra Bemba, Giovanniego Della Casa i innych [...] (IW1, p. 21)

(15) [...] triumfy święcił dojrzały barok w wydaniu Gianlorenza Berniniego, Pietra da Cortona i Francesca Borrominiego. (IW5, p. 100)

29 Si tratta sempre di una nota a pie di pagina; cf. nota 28.

30 Pietro da Cortona di cui si parla nel vol. 5 di IW (p. 100) è nato come Pietro Berrettini, per l'appunto, a Cortona; invece Giovanni Della Casa (IW1, p. 21) è nato in a Borgo San Lorenzo nel Mugello da Pandolfo in una villa detta "La Casa". 


\subsection{Cognomi Eco e Cau}

Il cognome Eco, nei casi dipendenti, ricorre 32 volte, tra cui una sola volta in forma declinata al gen. Eca (IW1, p. 76). Lo stesso cognome invariato non è sempre preceduto dal nome, ma la sintassi del periodo permette facilmente di riconoscere la sua funzione logica (p. es. tramite una preposizione), come in (16).

(16) Dwa przykłady cytowane przez Eco uświadomią nam jednak z całą mocą, czym jest [...] (IW1, p. 108)

L'autrice del testo in cui appare la forma $E c a$, a quanto pare, ha adoperato una strategia ben precisa: tutti i cognomi variabili riferiti nel saggio sono declinati (32 occorrenze testuali di 4 cognomi: Cambelotti, D'Annunzio, Leoni, Michetti), tranne Eco che, però, rimane invariato se accompagnato dal nome (l'autrice rispetta, quindi, pienamente i consigli grammaticali che abbiamo visto prima; si vedano gli esempi 17-18). Similmente, il cognome invariabile $B o$ è stato preceduto dal nome in forma declinata (19).

(17) Łatwo to wyjaśnić, odwołując się do koncepcji Umberta Eco, zgodnie z którą każdy tekst [...] (IW1, p. 67)

(18) [...] wróćmy na chwilę do przemyśleń Borgesa i Eca dotyczących natury tekstu literackiego jako tworu niedefinitywnego [...] (ivi, p. 76)

(19) Italo Calvino, komentując słowa Carla Bo, że [...] (ivi, p. 68)

Nel testo interamente dedicato all'autore de Il nome della rosa si preferiscono le forme invariate (tutti gli altri cognomi variabili vengono declinati: Baricco, Calvino, Camilleri, Carmi, Carti, Dorfles, Gramsci, Moravia, Pasolini, Pincherli, Pitigrilli, Segre, Tabucchi, Tasso); il cognome invariabile $F o$ viene preceduto dal nome: [...] nagrody Nobla dla Daria Fo (IW7, p. 183).

Tra le forme invariate troviamo anche il cognome Cau che potrebbe appartenere a due categorie descritte nella sezione 2.3: a) cognomi uscenti in $-u$, quindi invariabili, o b) cognomi uscenti in -au, di cui non si declina solo la forma del locativo. L'autrice del testo ha lasciato il det- 
to cognome invariato, in due frasi preceduto dal nome (20-21), in altre due inserito in un sintagma coordinato con altro cognome - a sua volta declinato - il cui caso indica la funzione logica dell'insieme (22-23).

(20) $[\ldots]$ pracujący $\mathrm{w}$ tandemie translatorskim z Giovannim Cau (IW1, p. 167)

(21) Wraz z zaprzyjaźnionym Giovannim Cau Tłuchowski przełożył cztery wiersze [...] (ivi, p. 168)

(22) W metrycznych wersjach Cau i Skarbka-Tłuchowskiego pojawiają się często rymy gramatyczne (21) [...] (ibidem)

(23) Rozważając wartość przekładów Cau i Skarbka-Tłuchowskiego [...] (ibidem)

\subsection{Eventuale incoerenza flessiva e strategie degli autori}

In un numero relativamente alto di testi in cui appaiono le forme invariate (5 su 10) è facile riconoscere una strategia condivisa dagli autori: tutti i cognomi in un dato testo vengono declinati tranne quelli preceduti da una preposizione (che sono accuratamente preceduti dal nome). Tale strategia è stata adoperata nei seguenti testi a) IW1, pp. 18-36, dove troviamo 14 forme declinate di cognomi semplici (Achillini, Bembo, Bruno, Colonna, Fiamma, Marino, Preti, Tasso) e 3 forme non declinate di cognomi preposizionali da Barberino, Della Casa, da Tempo (i primi due indicano, effettivamente, la provenienza); b) IW5, pp. 107-121, con 9 forme declinate (dei cognomi Bernini, Borromini, Ferrata, Guarini, Guidi, Scianzi) e una sola non declinata, de Carlis; c) IW5, pp. 317-337, con 1 forma declinata (Pertillo) e 1 forma invariata ( $d a$ Ponte; è variabile perché in questo caso non si tratta di un soprannome ma di un vero e proprio cognome); $d$ ) IW6, pp. 95-11, con 19 forme declinate (di Bembo, Gabriel, Carrera, Fazello, Maurolico) e 2 invariate di da Borgo San Sepolcro (quindi un soprannome); e) IW7, pp. 87-103; con 37 forme declinate (Rosmini, Gentile, Manzoni, Petronio, Rosmini, Sapegno, Varese, Viti) e 1 forma invariata di De Sanctis.

Altre strategie adoperate da un singolo autore sono: $a$ ) la mancata declinazione di tutte le forme flesse di un certo cognome scelto tra vari cognomi che ricorrono nel testo: IW6, pp. 253-284, dove 17 
cognomi vengono declinati ${ }^{31}$ e 2 rimangono sempre invariati: $D a$ Feltre (soprannome giustamente invariato) e Lombroso (facilmente declinabile; ricorre anche senza il nome); $b$ ) la mancata declinazione dei cognomi appartenenti a un dato modello flessivo: IW3, pp. 65-84, dove 62 forme sono declinate ${ }^{32}, 5$ occorrenze rimangono invariate, tra cui da Todi (soprannome) e due cognomi uscenti in -e, Felice e Pisacane (nel testo non ricorrono altri cognomi di questo gruppo).

Non mancano - chiaramente - piccole sviste redazionali, p. es. IW7, pp. 145-161, dove tra le 19 forme diverse dal nominativo (di 5 cognomi Cellini, Gozzano, Neri, Svevo, Pavese) solo 1 forma è rimasta invariata (gen. Pavese; altre 12 occorrenze dello stesso cognome sono state declinate: gen. Pavesego), oppure la mancata declinazione di alcuni cognomi preceduti da un'iniziale del nome nelle note a pie di pagina (IW1, pp. 50-62; cf. sopra).

Il meno coerente dal punto di vista flessivo è il testo IW1, pp. 13-23, dove su 7 occorrenze diverse dal nominativo troviamo 1 cognome invariato (gen. Garrone), 1 cognome declinato (gen. Pasoliniego < nom. Pasolini), e 1 cognome le cui forme sono sia declinate che invariate (gen. Saviana o Saviano, acc. Saviana; le forme invariate non sono accompagnate dai nomi).

\section{CONLUSIONI}

Come abbiamo visto, la morfologia flessiva polacca è particolarmente complessa e il numero di regole riguardanti la declinazione dei cognomi stranieri può essere scoraggiante, soprattutto per chi apprende la lingua

${ }^{31}$ Sono in totale 26 le occorrenze dei seguenti cognomi appartenenti a tutti i modelli flessivi: Aretino, Boccaccio, Campanella, Codignoli, Collodi, Colonna, Croce, De Amicis, Fanciulli, Frescobaldi, Gentile, Lombardo Radice, Mussolini, Piccolomini, Pitigrilli, Turiello.

32 Sono addirittura 35 cognomi: Aleardi, Alfieri, Berchet, Bontempelli, Buzzi, Callagari, Carducci, Collodi, Dall'Ongaro, D'Annunzio, Foscolo, Garibaldi, Giusti, Leopardi, Macchiavelli, Mameli, Manzoni, Mazzini, Mercantini, Monti, Nardini, Niccolini, Nievo, Palazzeschi, Papini, Pascoli, Pellico, Quasimodo, Ricciardi, Rossetti, Soffici, Stecchetti, Tasso, Verga, Zanella. 
polacca (in più, nelle precedenti pagine abbiamo visto soltanto le norme relative ai cognomi italiani). Tali regole dovrebbero, pertanto, essere definite in maniera possibilmente più precisa per non dare adito ad alcun dubbio di natura flessiva. Nei dizionari ortografici e in altre fonti di relative regole, andrebbe detto expressis verbis, a titolo d'esempio, che a) i cognomi uscenti i $-c a$, similmente a quelli in -co, cambiano dov'è necessario - l'ortografia: nom. - $c a>$ gen. $-k i$, come nom. $-c o>$ -co > str. -kiem (viene citato soprattutto il secondo caso), b) l'invariabilità dei cognomi uscenti in [wa] riguarda soltanto i cognomi francesi; simili cognomi italiani andrebbero declinati, $c$ ) i cognomi in -cia, -gia, -scia raddoppiano la vocale $i$ davanti alla medesima desinenza $-i$, quindi gen./dat./loc. -cii, -gii, -scii (andrebbero, in questo caso, aggiunti anche gli esempi dei cognomi terminanti in -ia, dove la vocale $i$ è accentata, p. es. nom, Beccaria, gen./dat./loc. Beccarii).

Sono particolarmente insidiosi i cognomi introdotti da preposizioni, visto che la loro flessione richiede inoltre certe conoscenze extralinguistiche: qualora non si tratti di un vero cognome ma di un soprannome, questo non va declinato. Tuttavia, non è sempre facile riconoscere la vera natura del nome proprio che si deve affrontare, per così dire, nella stesura del testo (p. es. Wolański, 2018, indica la giusta declinazione del cognome da Bologna: gen. da Bologni ecc.; ma nel caso di Jacopo da Bologna - vale a dire Jacopino di Francesco de' Bavosi - si tratta, effettivamente, di un soprannome). Inoltre, le norme polacche parlano solo dei cognomi introdotti dalla preposizione $d a$ (cf. Wolański, 2013, 2018), come $d a$ Vin$c i$, da Cortona (cf. anche taBella 3). In effetti, tali nomi propri indicano spesso la provenienza del personaggio cui sono stati attribuiti. Ciononostante, tra i nomi propri italiani troveremo varie forme introdotte da altre preposizioni, p. es. di o del, che sono - anch'esse - soprannomi e che possono facilmente essere trattate come cognomi veri e propri (ricordiamo, a titolo d'esempio, Adrea del Sarto, vale a dire Andrea d'Agnolo di Francesco di Luca, pittore rinascimentale, figlio - per l'appunto - di un sarto cui deve il suo soprannome, o Piero di Cosimo, quindi Piero di Lorenzo di Piero d'Antonio, che deve il soprannome a Cosimo Rosselli nella cui bottega ha svolto l'apprendistato). Di tali cognomi (sopranno$\mathrm{mi})$, nelle norme polacche, non c'è alcuna traccia. 
Abbiamo anche visto che, nei testi polacchi apparsi su Italica Wratislaviensia, sebbene la maggior parte dei cognomi sia stata accuratamente declinata, non mancano casi in cui gli autori hanno preferito conservare la forma invariata, che è, di conseguenza, preceduta dal nome del personaggio (non mancano neppure - anche se poche - forme invariate laddove non dovevano ricorrere). Come si è detto prima, è una strategia accettabile per i nomi uscenti in $-o$ ed $-e$ che, però, può rendere il testo meno scorrevole, soprattutto perché sarebbe inutile, dal punto di vista informativo, ripetere il nome davanti a ogni occorrenza di un dato cognome, specie quando le sue occorrenze sono numerose.

La scelta grammaticale e stilistica consigliata dall'autore del presente testo è di declinare tutti i cognomi italiani variabili tranne quelli che terminano con le seguenti vocali accentate: $-\grave{a},-\grave{e}$, $-\grave{i}$ (i cognomi che terminano in -ò e -ù rimangono comunque invariati, secondo le norme polacche). Simili cognomi andrebbero preceduti dal nome del loro portatore in forma declinata. In tal modo sarà possibile riconoscere facilmente sia la funzione logica del sintagma sia la forma del nominativo del cognome. Per i nomi preceduti dalla preposizione $d a$, nel caso in cui risulta difficile stabilire se si tratti di un vero cognome e un soprannome, si consiglia di lasciare la forma invariata, che va sempre preceduta da un nome comune attribuito al personaggio o dal suo primo nome. Ogni eventuale incoerenza flessiva all'interno dello stesso testo è stilisticamente scorretta.

\section{BIBLIOGRAFIA}

Alinei, M., \& Benozzo, F. (2017). Dizionario etimologico-semantico dei cognomi italiani. Savona: PM.

Bajerowa, I. (1998). Wstyd nie odmieniać nazwisk. Poradnik Językowy, 10/1988, 51.

Bartmińska, I., \& Bartmiński, J. (1992). Stownik wymowy i odmiany nazwisk obcych. Olsztyn: Książnica Polska.

Broniś, O. (2017). Analiza semantyczna i fonetyczna wybranych fałszywych przyjaciół polsko-włoskich. In M.B. Majewska (Ed.), Wokół homonimii międzyjęzykowej (pp. 175-192). Warszawa: UKSW. 
Cieślikowa, A. (2008). Maty stownik odmiany nazw własnych (2nd ed.). Warszawa: Rytm.

De Felice, E. (1978). Dizionario dei cognomi italiani. Milano: Mondadori.

Gruszczyński, W., \& Bralczyk, J. (2002). Stownik gramatyki języka polskiego. Warszawa: WSiP.

Grzenia, J. (2002). Stownik nazw własnych. Warszawa: PWN.

Grzenia, J. (2007, March 30). Charles Pasqua. Poradnia językowa PWN. Retrieved from sjp.pwn.pl/poradnia/haslo/Charles-Pasqua;8110.html.

Jadacka, H. (2005). Kultura języka polskiego. Fleksja, słowotwórstwo, składnia. Warszawa: PWN.

Karaś, M., \& Madejowa, M. (Eds.). (1977). Słownik wymowy polskiej PWN. Warszawa/Kraków: PWN.

Katedra Kulturoznawstwa DSW (n.d.). Poradnia językowa Dolnoślaskiej Szkoty Wyższej. Retrieved from dsw.edu.pl/kulturoznawstwo/kultura-od-salonu/poradnia-jezykowa.

Konik, R. (2003). Katastrofiści i przystosowani czyli typologia współczesnych intelektualistów według Umberta Eca. Nowa Krytyka, 14, 269-279.

Kwapisz-Osadnik, K. (2012). Podstawowe wiadomości z gramatyki polskiej $i$ włoskiej. Szkic porównawczy. Katowice: UŚ.

Liotta, R. (1992). Lingua polacca. II. Tavole sinottiche. Milano: Vita e Pensiero.

Markowski, A. (Ed.). (2006). Wielki stownik poprawnej polszczyzny. Warszawa: PWN.

Maturi, P. (2006). I suoni delle lingue, i suoni dell'italiano. Introduzione alla fonetica. Bologna. il Mulino.

Miodunka, W. (1977). Pisownia fonetyczna w słowniku wymowy polskiej. In M. Karaś \& M. Madejowa (Eds.), Słownik wymowy polskiej PWN (pp. xxiv-xxvi). Warszawa/Kraków: PWN.

Nosilia, V. (2017). Il sostantivo. In eadem (Ed.), Polacco compatto. Dizionario polacco/italiano, italiano/polacco (pp. 477-484). Bologna: Zanichelli.

Ostaszewska, D., \& Tambor, J. (2004). Fonetyka i fonologia wspótczesnego języka polskiego. Warszawa: PWN.

Polański, E. (2016). Pisownia nazw własnych. In idem (Ed.), Wielki słownik ortograficzny PWN z zasadami pisowni i interpunkcji (pp. 93-108). Warszawa: PWN.

Polański, K. (Ed.). (2003). Encyklopedia językoznawstwa ogólnego. Wrocław/ Warszawa/Kraków: Ossolineum. 
Wolańki, A. (2013, October 7). Pietro da Cortona. Poradnia językowa PWN. Retrieved from sjp.pwn.pl/poradnia/haslo/;14527.

Wolańki, A. (2018, April 16). Odmiana nazwisk obcych z przyimkami. Poradnia językowa PWN. Retrieved from sjp.pwn.pl/poradnia/haslo/Odmiana-nazwisk-obcych-z-przyimkami; 18537.html.

Wojtowicz, W. (1997). Umberto Eco i światy możliwe. Pamiętnik Literacki, 88/2, 77-96.

Zdunkiewicz-Jedynak, D. (2006). Nazwiska [hasło problemowe]. In A. Markowski (Ed.). (2006), Wielki stownik poprawnej polszczyzny (pp. 1612-1623). Warszawa: PWN.

\section{APPENDICE}

TABELLA 4: Cognomi italiani che ricorrono nei testi polacchi pubblicati su IW

Statistica generale: occorrenze: 743; lemmi: 234; gen.: 611 occorrenze; dat.: 9; acc.: 76; str.: 26; loc.: 21 Cognomi femminili (72 occorenze in totale; 14 lemmi)

- variabili (18 occorrenze; 3 lemmi): Colonna, Flora, Venezia

- invariabili (54 occorrenze; 11 lemmi): Agus, Carti, Giusti, Frescobaldi, Ginzburg, Leoni, Masoero, Mazzucco, Montessori, Orlandi, Viganò

Cognomi maschili (671 occorrenze; 220 lemmi)

- uscenti in -i (109 lemmi): Achillini, Alberti, Albinioni, Aleardi, Alfieri, Ballerini, Bastiani, Benedetti, Benni, Benzoni, Berlusconi, Bernini, Bianchi, Bioni, Boccardi, Bontempelli, Borromini, Braccioli, Brunetti, Burigotti, Buzzi, Callagari, Cambelotti, Camilleri, Caprotti, Carducci, Carmi, Casparini, Castaldi, Cellini, Codignoli, Coletti, Collodi, Conti, Fanciulli, Fanfani, Finazzi, Garibaldi, Giusti, Gramsci, Guarini, Guccini, Guidi, Lampugnani, Leopardi, Luchini, Macchiavelli, Mameli, Mancini, Manzoni, Marconi, Mareschi, Marescotti, Martinelli, Mazzini, Melani, Mercantini, Michetti, Mingotti, Monteverdi, Monti, Moretti, Mussolini, Nardini, Negretti, Neri, Niccolini, Orlandi, Paganini, Palazzeschi, Papini, Pariatti, Pascoli, Pasolini, Patricelli, Pavolini, Perini, Peruzzi, Pesserini, Piccolomini, Pincherli, Pitigrilli, Preti, Regreti, Ricci, Ricciardi, Roncalli, Rosmini, Rossetti, Rossi, Salaì, Salgari, Salvi, Santi, Scalabrini, Scianzi, Sensini, Silvani, Simonetti, Soffici, Sorti, Stecchetti, Storni, Tabucchi, Trevisani, Vettori, Viti, Vittorini, Vivaldi

- uscenti in -o (40 lemmi): Aretino, Baricco, Bellarmino, Beltramo, Bembo, Berto, Boccaccio, Bronzino, Bruno, Calvino, Canaletto, Denzio, Eco, Fazello, Fogazzaro, Foscolo, Gozzano, Lombroso, Mariano, Marino, Maurolico, Metastasio, Nievo, Nullo, Paisiello, Parrio, Pellico, Pertillo, Petronio, Pirandello, Pozzo, Quaglio, Quasimodo, Ripellino, Sapegno, Saviano, Svevo, Tasso, Turiello, Zeno 
- uscenti in - $\boldsymbol{a}$ (21 lemmi): Cadorna, Caldara, Campanella, Carrera, Casanova, Colonna, Drissina, Ferrata, Fiamma, Gadda, Jossa, La Capria, Mantegazza, Moravia, Murgia, Piva, Porta, Sciascia, Verga, Vida, Zanella

- uscenti in -e (14 lemmi): Bonaparte, Carlone, Croce, Errante, Felice, Garrone, Gentile, Malaparte, Montale, Pavese, Pisacane, Provisore, Segre, Varese

- terminano con una consonante (13 lemmi): Berchet, Canal, Cavour, Cordans, Cortis, Dorfles, Dreyer, Gabriel, Madonis, Magris, Niuron, Odenig, Tilgher

- invariabili (3 lemmi): Bo, Fo (Cau)

- preceduti da una preposizione (18 lemmi): da Barberino, da Borgo San Sepolcro, da Cortona, da Feltre, da Parmataro, da Ponte, da Tempo, da Todi, da Vinci, Dall'Ongaro, D’Annunzio, De Amicis, de Carlis, de Carlo, De Gasperi, De Sanctis, della Bella, Della Casa

- doppi (2 lemmi): Lombardo Radice, Tomasi di Lampedusa

Riassunto: La morfologia flessiva polacca, con il suo ricco inventario di regole relative alla declinazione sostantivale, coinvolge ugualmente i cognomi di provenienza straniera. In linea di massima, simili cognomi andrebbero declinati come nomi comuni o aggettivi polacchi, a seconda del paradigma flessivo cui appartengono. In realtà, la declinazione dei sostantivi stranieri comporta vari problemi di natura fonetica, ortografica e, soprattutto, flessiva, non solo per via di un numero relativamente alto di regole che governano il sistema morfologico polacco, ma anche perché alcune regole non sono state determinate in maniera definitiva e la stessa flessione, in alcuni casi, non è obbligatoria. In effetti, gli utenti della lingua polacca, per evitare di declinare un dato cognome, preferiscono spesso ricorrere a varie strategie redazionali. Nei loro testi non mancano, però, né forme sbagliate dei cognomi, né strategie flessive incoerenti o addirittura scorrette. Con il presente lavoro si intende, quindi, esporre in maniera possibilmente più dettagliata le regole relative alla declinazione dei cognomi italiani in lingua polacca insieme all'analisi delle occorrenze di tali cognomi nei testi polacchi apparsi su Italica Wratislaviensia.

Parole chiave: flessione sostantivale, declinazione, nomi propri, cognomi, cognomi italiani in polacco 